\title{
GMR
}

\section{Associations between HIF-1a polymorphisms C1772T and G1790A and susceptibility to chronic obstructive pulmonary disease}

\author{
W.T. Wei' ${ }^{1}$ B. $\mathrm{Li}^{2}$, M. Chen ${ }^{3}$, H.R. Jia ${ }^{3}$ and H.X. Zhang ${ }^{3}$ \\ 'Department of General Medicine, Guangdong General Hospital, \\ Guangdong Academy of Medical Sciences, Guangzhou, China \\ ${ }^{2} \mathrm{ICU}$, Longgang Central Hospital of Shenzhen City, Shenzhen, China \\ ${ }^{3} \mathrm{ICU}$, Guangdong General Hospital, Guangdong Academy of Medical Sciences, \\ Guangzhou, China \\ Corresponding author: H.X. Zhang \\ E-mail: zhanghxmedsci@126.com
}

Genet. Mol. Res. 14 (4): 17341-17347 (2015)

Received August 11, 2015

Accepted October 1, 2015

Published December 21, 2015

DOI http://dx.doi.org/10.4238/2015.December.21.2

\begin{abstract}
In the present study, we examined whether hypoxia-inducible factor-1 $\alpha$ (HIF-1 $\alpha)$ polymorphisms are associated with the susceptibility to chronic obstructive pulmonary disease (COPD). One hundred and twenty patients with COPD and 112 healthy controls were recruited from the Han population in Southern China. Polymerase chain reaction-restriction fragment length polymorphism was used to assess the C1772T and G1790A polymorphisms in the HIF-1 $\alpha$ gene, and differences in genotypes between the 2 groups were compared. No significant difference in the distribution of the C1772T polymorphism was observed between COPD cases and healthy controls $(P>0.05)$. In contrast, the distribution of the G1790A polymorphism was significantly different between the 2 groups ( $P$ $<0.05$ ). Our results indicated that the HIF-1a G1790A polymorphism was
\end{abstract}


associated with an increased susceptibility to COPD in Han subjects from Southern China.

Key words: Chronic obstructive pulmonary disease; Hypoxia-inducible factor-1 $\alpha$; Single nucleotide polymorphisms

\section{INTRODUCTION}

Chronic obstructive pulmonary disease (COPD) is a progressive disease characterized by chronically poor airflow. It is closely related to chronic bronchitis and emphysema and may severely affect the quality of life of some patients. Over the past several years, both the incidence and mortality of COPD have increased (Pauwels et al., 2001; Viegi et al., 2001; Wouters, 2003). Cigarette smoking is regarded as the most important environmental factor, and approximately $85 \%$ of COPD patients are long-term smokers. However, only $10-20 \%$ of smokers develop symptomatic COPD. Moreover, individual differences are typically observed for smoking-induced airway obstruction (Burrows et al., 1977), suggesting that a patient's individual characteristic may be associated with COPD phenotypes. Previous studies found an increased frequency of COPD within families, suggesting that in addition to environmental factors, genetic factors may also play important roles in disease development and progression.

Accumulating evidence has demonstrated that COPD is not just a chronic pulmonary disease, but also a chronic systemic inflammation syndrome (Fabbri and Rabe, 2007). Several types of inflammatory cells and mediators affect the airway and pulmonary parenchyma, thus promoting COPD development and progression (Liebhart et al., 2005; Hersh et al., 2006; Homma et al., 2006; Fu et al., 2007a,b; Keicho and Matsushita, 2007). Hypoxia-inducible factor-1 (HIF-1) is a transcription factor in mammalian cells that mediates the adaptive response to changes in cellular oxygen levels. HIF-1 consists of 2 subunits: the oxygen-regulated HIF-1 $\alpha$ subunit ( $120 \mathrm{kDa}$ ) and the constitutively expressed HIF-1 $\beta$ subunit (91-94 kDa). The transcriptional activity of HIF-1 is primarily regulated by the expression and stability of HIF-1 $\alpha$, which are associated with hypoxia adaptation, expression of multiple inflammatory factors, and immune responses. HIF-1 expression is increased in tissues and cells under hypoxic conditions. This promotes the expression of several hypoxia-related genes involved in the adaptive physiological response to these environmental changes. HIF-1 also plays an important role in the expression of genes involved in inflammation, energy metabolism, angiogenesis, and airway remodeling (Fu et al., 2005). The role of HIF in the development and progression of COPD has also been demonstrated. For instance, previous studies showed that HIF-1 plays an important role in critical pathological processes of pulmonary heart disease in patients with hypoxic pulmonary hypertension and COPD (Earley and Resta, 2002; Manalo et al., 2005; Ohsawa et al., 2005; Semenza, 2005; He et al., 2006; Kong and Dai, 2006; Schultz et al., 2006).

Single nucleotide polymorphisms (SNPs) are single-base substitutions or transversions that occur in more than $1 \%$ of the entire population (Mullikin et al., 2000). SNPs within coding regions may result in changes to the amino acid sequence, and such changes may significantly alter the activity and biological properties of the encoded protein. C1772T and G1790A are 2 common SNPs within HIF-1 1 that result in a proline to serine change at position 582 and an alanine to threonine change at position 588, respectively. Amino acid changes at these 2 positions may affect the oxygen-dependent degradation of HIF-1 $\alpha$ as well as influence the stability and transcriptional activity of the protein. As a result, regulation of downstream target genes may also be affected (Li et al., 2004; Fu et al., 2005; Koukourakis et al., 2006). 
Previous studies have reported that serum levels of HIF-1 $\alpha$ are significantly higher in COPD patients compared to individuals without the disease. However, few studies have investigated the association between HIF-1a polymorphisms and COPD susceptibility. In the present study, we examined the potential associations between HIF-1a polymorphisms and the susceptibility to COPD to clarify the role of HIF-1a in the pathogenesis of the disease and to provide a foundation for the development of new options for treating patients with COPD.

\section{MATERIAL AND METHODS}

\section{Subjects}

One hundred and twenty COPD patients that had been treated in the Department of Respiration of Guangdong Provincial People's Hospital were included in the case group. One hundred and twelve age- and gender-matched healthy subjects that received physical examinations in the Physical Examination Center of Guangdong Provincial People's Hospital during the same time period were included in the control group.

Patients were diagnosed with COPD according to the Global Initiative for Chronic Obstructive Lung Disease (GOLD), had no obvious occupational exposures, and volunteered to participate in the study. Patients with other diseases that could damage pulmonary function, including asthma and pulmonary fibrosis, were excluded.

COPD was diagnosed according to the GOLD criteria issued in 2007. For all patients, medical records were reviewed and physical examinations, including forced expiratory volume in first second (FEV1)/forced vital capacity (FVC) and FEV1/expected value (\%), were performed. Additionally, plain chest $\mathrm{X}$-rays or high-resolution chest computed tomography scanning were performed to diagnose COPD (FEV1/FVC $<70 \%$ and FEV1/expected value $\%<80 \%$ ).

All subjects included in the study were unrelated and of Han nationality from Northern China. No genetic interference from other ethnicities (i.e., marriage with people from other ethnicities) was observed. Written informed consent was obtained from all study participants. This study was approved by the Ethics Committee of Guangdong Provincial People's Hospital (2009076).

\section{Blood collection and genotyping}

For each subject in both the case and control groups, $4 \mathrm{~mL}$ anticoagulant peripheral venous blood was collected. Genomic DNA was extracted from peripheral blood mononuclear cells using kits from Tiangen Biochemical Technology Co., Ltd. (Beijing, China) and stored at $-20^{\circ} \mathrm{C}$ until use.

C1772T and G1790A polymorphisms of HIF-1a were genotyped by polymerase chain reaction-restriction fragment length polymorphism according to previously described methods (Hersh et al., 2006). For genotyping of the C1772T polymorphism, the forward primer was 5'-AAGGTGTGGC CATTGTAAAAACTC-3' and the reverse primer was 5'-GCACTAGTAGTTTCTTTATGTATG-3'. For genotyping of the G1790A polymorphism, the forward primer was 5'- AAGGTGTGGCCATTGTAAA AA-3' and the reverse primer was 5'-TGACTCAAAGCGACAGATAACACG-3'. Sequencing of the polymerase chain reaction products was also performed.

\section{Statistical analysis}

The SPSS 13.0 software was used for statistical analysis (SPSS, Inc., Chicago, IL, USA). 
The mean and standard deviation were used to describe quantitative data with normal distribution. Hardy-Weinberg equilibrium was tested to evaluate the representation of each genotype. The chi-square test was used to compare the frequencies of different genotypes, allele distribution, and gender between the 2 groups, and the $t$-test was used to compare age, smoking index, and pulmonary function (FEV1/FVC and FEV1/expected value\%) between the 2 groups. $\mathrm{P}<0.05$ was considered to be statistically significant.

\section{RESULTS}

\section{General characteristics of the subjects}

The mean age of the 112 age- and gender-matched controls (95 males and 17 females) was 67 years (40-81 years). Medical records, physical examinations, and chest X-rays of these subjects suggested no diseases of the bronchus, lungs, or heart. Moreover, pulmonary function tests showed that the FEV1/FVC was $>70 \%$ and FEV1/expected value $\%$ was $>80 \%$. No significant differences in gender, age, or smoking index were found between cases and controls. However, the FEV1/expected value\% and FEV1/FVC (\%) were significantly higher in the controls than in the cases (Tables 1 and 2).

Table 1. Clinical characteristics of subjects in the case and control groups.

\begin{tabular}{lccr}
\hline & Cases $(\mathrm{N}=120)$ & Controls $(\mathrm{N}=112)$ & $P$ value \\
\hline Gender (male:female) & $105: 15$ & $95: 1$ & 0.5544 \\
Age (years) & $67.72 \pm 10.5(40-95)$ & $67.8 \pm 7.6(40-81)$ & 0.9510 \\
Smoking index (year*number of cigarettes), both $>300$ & $381 \pm 406$ & $377 \pm 510$ & 0.9502 \\
FEV1/ FVC & $58.2 \pm 5.9(<70 \%)$ & $96.8 \pm 12.8(>70 \%)$ & $<0.0001$ \\
FEV1/expected value\% & $65.7 \pm 7.1$ & $86.4 \pm 11.0$ & $<0.0001$ \\
\hline
\end{tabular}

Table 2. Hardy-Weinberg equilibrium of HIF-1 $1 \alpha$ C1772T/G1790A in the two groups.

\begin{tabular}{|c|c|c|c|c|c|c|c|c|}
\hline & \multicolumn{4}{|c|}{$\mathrm{C} 1772 \mathrm{~T}$} & \multicolumn{4}{|c|}{ G1790A } \\
\hline & $\mathrm{CC}$ & CT & TT & $P$ value & GG & GA & AA & $P$ value \\
\hline Cases & 105 & 15 & 0 & 0.465 & 116 & 4 & 0 & 0.853 \\
\hline Controls & 105 & 7 & 0 & 0.733 & 101 & 11 & 0 & 0.585 \\
\hline Total & 210 & 22 & 0 & 0.448 & 217 & 15 & 0 & 0.611 \\
\hline
\end{tabular}

\section{Hardy-Weinberg equilibrium}

As shown in Table 2, the genotype distribution of HIF-1a was in Hardy-Weinberg equilibrium for cases, controls, and all subjects included. This suggests that the distribution of HIF$1 \alpha$ genotypes in the subjects included was representative of the genotype distribution in the entire population.

\section{Distribution of the C1772T polymorphism}

The frequencies of the CC, CT, and TT genotypes of the C1772T polymorphism in exon 12 of HIF-1 1 were $87.5,12.5$, and $0 \%$, respectively, in the 120 cases of COPD; these values were $93.8,6.2$, and $0 \%$, respectively, in the controls. There were no significant differences in genotype 
frequencies between the 2 groups ( $P>0.05$ ). A total of 93.8 and $6.2 \%$ of patients with COPD carried the $\mathrm{C}$ and $\mathrm{T}$ alleles, respectively. Analysis of healthy controls showed that 96.9 and $3.1 \%$ of individuals in this group carried the $\mathrm{C}$ and $\mathrm{T}$ alleles, respectively. No significant difference in allele distribution was found between the 2 groups ( $P>0.05$; Table 3$)$.

Table 3. Distribution of alleles and genotypes of HIF-1a C1772T in the two groups.

\begin{tabular}{llccrrr}
\hline & $N$ & \multicolumn{3}{c}{ Genotypes } & \multicolumn{2}{c}{ Alleles } \\
\cline { 3 - 5 } & & CC & CT & TT & C \\
\hline Cases & 120 & $105(87.5 \%)$ & $15(12.5 \%)$ & $0(0 \%)$ & $225(93.8 \%)$ & $15(6.2 \%)$ \\
Controls & 112 & $105(93.8 \%)$ & $7(6.2 \%)$ & $0(0 \%)$ & $217(96.9 \%)$ & $7(3.1 \%)$ \\
\hline
\end{tabular}

Chi-square test result: cases vs controls, $\mathrm{P}=0.1044$.

\section{Distribution of the G1790A polymorphism}

The frequencies of the GG, GA, and AA genotypes of the G1790A polymorphism in exon 12 of HIF-1 $\alpha$ were $96.7,3.3$, and $0 \%$, respectively, in the 120 COPD cases; these values were 90.2, 9.8 , and $0 \%$, respectively, in the controls. The difference in the distribution of different genotypes was statistically significant between the 2 groups $(P<0.05)$. A total of 98.4 and $1.6 \%$ of patients with COPD carried the $\mathrm{G}$ and A alleles, respectively; in contrast, 95.1 and $4.9 \%$ of healthy controls carried the $G$ and $A$ alleles, respectively. The difference between the 2 groups was statistically significant $(P<0.05$; Table 4).

Table 4. Distribution of alleles and genotypes of HIF-1a G1790A in the two groups.

\begin{tabular}{|c|c|c|c|c|c|c|}
\hline & \multirow[t]{2}{*}{$\mathrm{N}$} & \multicolumn{3}{|c|}{ Genotypes } & \multicolumn{2}{|c|}{ Alleles } \\
\hline & & GG & GA & AA & G & A \\
\hline Cases & 120 & $116(96.7 \%)$ & $4(3.3 \%)$ & $0(0 \%)$ & $236(98.4 \%)$ & $4(1.6 \%)$ \\
\hline Controls & 112 & $101(90.2 \%)$ & $11(9.8 \%)$ & $0(0 \%)$ & $213(95.1 \%)$ & 11 (4.9\%) \\
\hline
\end{tabular}

Chi-square test result: cases vs controls, $\mathrm{P}=0.0446$.

\section{DISCUSSION}

In recent years, HIF-1a has emerged as a key factor in COPD. In the present study, HIF1a polymorphisms were assessed using the polymerase chain reaction-restriction fragment length polymorphism method, and the potential associations with COPD susceptibility were investigated. We found that the distribution of different genotypes and alleles of the HIF-1a C1772T polymorphism was not significantly different between cases and controls. In contrast, the distribution of different genotypes and alleles of the HIF-1a G1790A polymorphism was statistically different between the 2 groups. These findings suggest that HIF-1a polymorphisms are closely associated with COPD development.

HIF-1 critically mediates the adaptive response to hypoxia. Previous studies have demonstrated that HIF-1a polymorphisms show statistically significant differences regarding their involvement in the development and progression of multiple diseases (Tanimoto et al., 2003; Liu et al., 2007). Additionally, several other studies have shown that HIF-1a polymorphisms are significantly associated with several diseases (including cancer, diabetes, and coronary heart disease) in people of different ethnicities (Yamada et al., 2005; Hebert et al., 2006; Hlatky et al., 2007; Kim et al., 2008). The HIF-1a C1772T (P582S) and G1790A (A588T) polymorphisms may 
significantly elevate HIF-1a protein expression and lead to increased transcription of downstream target genes (Tanimoto et al., 2003; Fu et al., 2005; Koukourakis et al., 2006). A previous study showed that both mRNA and protein expression of HIF-1a were significantly decreased in the lung tissue of patients with severe COPD/emphysema (Yasuo et al., 2011). These studies suggest that the A allele at position 1790 of the HIF-1a gene could protect against COPD, while the $\mathrm{G}$ allele may be a risk factor for COPD. Additionally, the HIF-1a G1790A polymorphism may decrease COPD risk, which is consistent with our findings. No significant association between the C1772T polymorphism and COPD was found in the present study; however, whether there is a significant association between this polymorphism and COPD remains unclear. Previous studies identified an association between the HIF-1a C1772T polymorphism and prostate cancer; however, an association between the HIF-1 1 C $1772 \mathrm{~T}$ polymorphism and hypoxic biomarker expression has not been observed (Foley et al., 2009).

COPD is a chronic systemic inflammation syndrome characterized by irreversible, progressive airflow obstruction (Fabbri and Rabe, 2007). HIF-1a is a critical factor responsible for maintaining oxygen homeostasis under hypoxia conditions, and this transcription factor was found to be associated with the development and progression of COPD. Our results demonstrate that the HIF-1a G1790A polymorphism is a risk factor for COPD susceptibility; however, the exact mechanisms involved in the influence of the HIF-1a G1790A polymorphism on the development and progression of COPD remain unclear and additional studies are required.

\title{
Conflicts of interest
}

The authors declare no conflict of interest.

\section{ACKNOWLEDGMENTS}

\author{
(\#2009B030801247).
}

\section{REFERENCES}

Burrows B, Knudson RJ, Cline MG and Lebowitz MD (1977). Quantitative relationships between cigarette smoking and ventilatory function. Am. Rev. Respir. Dis. 115: 195-205.

Earley S and Resta TC (2002). Estradiol attenuates hypoxia-induced pulmonary endothelin-1 gene expression. Am. J. Physiol. Lung Cell. Mol. Physiol. 283: L86-L93.

Fabbri LM and Rabe KF (2007). From COPD to chronic systemic inflammatory syndrome? Lancet 370: 797-799.

Foley R, Marignol L, Thomas AZ, Cullen IM, et al. (2009). The HIF-1alpha C1772T polymorphism may be associated with susceptibility to clinically localised prostate cancer but not with elevated expression of hypoxic biomarkers. Cancer Biol. Ther. 8: 118-124.

Fu WP, Sun C, Dai LM, Yang LF, et al. (2007a). Relationship between COPD and polymorphisms of HOX-1 and mEPH in a Chinese population. Oncol. Rep. 17: 483-488.

Fu WP, Zhao ZH, Fang LZ, Sun C, et al. (2007b). Heme oxygenase-1 polymorphism associated with severity of chronic obstructive pulmonary disease. Chin. Med. J. 120: 12-16.

Fu XS, Choi E, Bubley GJ and Balk SP (2005). Identification of hypoxia-inducible factor-1alpha (HIF-1alpha) polymorphism as a mutation in prostate cancer that prevents normoxia-induced degradation. Prostate 63: 215-221.

$\mathrm{He} \mathrm{ZH}$, Zhang XF and Tan XW (2006). Changes of HIF-1a induced NOS expression during hypoxic pulmonary hypertension in rats. Chin. J. Tubere. Respir. Dis. 29: 2.

Hebert C, Norris K, Parashar P, Ord RA, et al. (2006). Hypoxia-inducible factor-1alpha polymorphisms and TSC1/2 mutations are complementary in head and neck cancers. Mol. Cancer 5: 3. 
Hersh CP, DeMeo DL, Raby BA, Litonjua AA, et al. (2006). Genetic linkage and association analysis of COPD-related traits on chromosome 8p. COPD 3: 189-194.

Hlatky MA, Quertermous T, Boothroyd DB, Priest JR, et al. (2007). Polymorphisms in hypoxia inducible factor 1 and the initial clinical presentation of coronary disease. Am. Heart J. 154: 1035-1042.

Homma S, Sakamoto T, Hegab AE, Saitoh W, et al. (2006). Association of phosphodiesterase 4D gene polymorphisms with chronic obstructive pulmonary disease: relationship to interleukin 13 gene polymorphism. Int. J. Mol. Med. 18: 933-939.

Keicho N and Matsushita I (2007). Progress in the genetics of chronic obstructive pulmonary disease. Nihon Rinsho 65: 611614.

Kim HO, Jo YH, Lee J, Lee SS, et al. (2008). The C1772T genetic polymorphism in human HIF-1alpha gene associates with expression of HIF-1alpha protein in breast cancer. Oncol. Rep. 20: 1181-1187.

Kong CC and Dai AG (2006). Expression of mitogen-actived protein kinase, phosphatidylinositol 3-kinase and hypoxiainducible factor-1alpha in pulmonary arteries of patients with chronic obstructive pulmonary disease. Zhonghua Jie He He Hu Xi Za Zhi 29: 372-375.

Koukourakis MI, Papazoglou D, Giatromanolaki A, Panagopoulos I, et al. (2006). C2028T polymorphism in exon 12 and dinucleotide repeat polymorphism in intron 13 of the HIF-1alpha gene define HIF-1alpha protein expression in non-small cell lung cancer. Lung Cancer 53: 257-262.

Li D, Hirsilä M, Koivunen P, Brenner MC, et al. (2004). Many amino acid substitutions in a hypoxia-inducible transcription factor (HIF)-1alpha-like peptide cause only minor changes in its hydroxylation by the HIF prolyl 4-hydroxylases: substitution of 3,4-dehydroproline or azetidine-2-carboxylic acid for the proline leads to a high rate of uncoupled 2-oxoglutarate decarboxylation. J. Biol. Chem. 279: 55051-55059.

Liebhart J, Polak M, Dobek R, Liebhart E, et al. (2005). TGF-beta1 gene polymorphism in chronic obstructive pulmonary disease. Pneumonol. Alergol. Pol. 73: 216-220.

Liu K, Sun X, Wang S and Hu B (2007). Association between polymorphisms of HIF-1alpha C1772T and G1790A and hypoxic acclimation in high altitude in Tibetans. Sheng Wu Yi Xue Gong Cheng Xue Za Zhi 24: 654-658.

Manalo DJ, Rowan A, Lavoie T, Natarajan L, et al. (2005). Transcriptional regulation of vascular endothelial cell responses to hypoxia by HIF-1. Blood 105: 659-669.

Mullikin JC, Hunt SE, Cole CG, Mortimore BJ, et al. (2000). An SNP map of human chromosome 22. Nature 407: 516-520.

Ohsawa S, Hamada S, Kakinuma Y, Yagi T, et al. (2005). Novel function of neuronal PAS domain protein 1 in erythropoietin expression in neuronal cells. J. Neurosci. Res. 79: 451-458.

Pauwels RA, Buist AS, Calverley PM, Jenkins CR, et al. (2001). Global strategy for the diagnosis, management, and prevention of chronic obstructive pulmonary disease. NHLBI/WHO Global Initiative for Chronic Obstructive Lung Disease (GOLD) Workshop summary. Am. J. Respir. Crit. Care Med. 163: 1256-1276.

Schultz K, Fanburg BL and Beasley D (2006). Hypoxia and hypoxia-inducible factor-1alpha promote growth factor-induced proliferation of human vascular smooth muscle cells. Am. J. Physiol. Heart Circ. Physiol. 290: H2528-H2534.

Semenza GL (2005). Involvement of hypoxia-inducible factor 1 in pulmonary pathophysiology. Chest 128: 592S-594S.

Tanimoto K, Yoshiga K, Eguchi H, Kaneyasu M, et al. (2003). Hypoxia-inducible factor-1alpha polymorphisms associated with enhanced transactivation capacity, implying clinical significance. Carcinogenesis 24: 1779-1783.

Viegi G, Scognamiglio A, Baldacci S, Pistelli F, et al. (2001). Epidemiology of chronic obstructive pulmonary disease (COPD). Respiration 68: 4-19.

Wouters EF (2003). Economic analysis of the Confronting COPD survey: an overview of results. Respir. Med. 97 (Suppl C): S3-S14.

Yamada N, Horikawa Y, Oda N, lizuka K, et al. (2005). Genetic variation in the hypoxia-inducible factor-1alpha gene is associated with type 2 diabetes in Japanese. J. Clin. Endocrinol. Metab. 90: 5841-5847.

Yasuo M, Mizuno S, Kraskauskas D, Bogaard HJ, et al. (2011). Hypoxia inducible factor-1alpha in human emphysema lung tissue. Eur. Respir. J. 37: 775-783. 